\title{
Analysis of Flow Field behind a Road Vehicle With/Without Rear Air Spoiler
}

\author{
Aly M. Elzahaby \\ Mechanical Power Engineering \\ Dept., Faculty of Engineering, \\ Tanta University, Tanta, Egypt
}

Mohamed I. Amro

Mechanical Power Engineering Dept., Faculty of Engineering,

Tanta University, Tanta, Egypt

\author{
El-Shenawy A. El-Shenawy \\ Mechanical Power Engineering \\ Dept., Faculty of Engineering, \\ Tanta University, Tanta, Egypt
}

Aly Hafez

Mechanical Power Engineering Dept., Faculty of Engineering, Tanta University, Tanta, Egypt
Abd-Elkader Said Bekhatro Mechanical Power Engineering Dept., Faculty of Engineering, Tanta University, Tanta, Egypt

Mohamed Adel Elmidany Mechanical Power Engineering Dept., Faculty of Engineering, Tanta University, Tanta, Egypt

\begin{abstract}
A particle image velocimetry (PIV) technique has been used to analyze the flow characteristics behind a road vehicle with/without an air spoiler attached on the behind end and also to estimate its effect on the wake. A vehicle model scaled in the ratio of 1/18 is set up in the mid-section of a closed-loop wind tunnel. The Reynolds number based on the vehicle length is taken in the range of $16.37 \times 103$ up to $3.77 \times 105$, i.e. $(V=(2$ to23.7 m/s). Measurements are carried out on the planes parallel to the free stream to investigate the two-dimensional structure of the flow field patterns on the vortices zone. There are significantly different vorticity distributions in the recirculation region; this depends upon the existence and height of the air spoiler. In general, the positions of the vortices are far away from the vehicle rear end due to the usage of the air spoiler. In addition, the levels of turbulence intensity and kinetic energy increases as the vehicle speed increases. The wing tip vortices generate the down force and as a result, they might make the vehicle more stable in driving.
\end{abstract} spoiler.

Keywords: PIV, road vehicle, Two-dimensional wake, Air

1. Introduction:

Many studies have been done to understand the flow pattern and wake structure behind a rear body of a road vehicle through numerical and experimental methods. For example the large eddy simulation (LES) method suggested the information of the three-dimensional wake structures, drag coefficients, as founded in Krajnovic and Davidson [1]. However, they have still shown lots of differences from the experimental data because the complicated geometry of a road vehicle and the high Reynolds number made the results sensitive to the number of mesh and so on. On the other hand, experiments on a full-size vehicle model can provide relatively exact information of the flow field. Most of the previous experimental works devoted to understand the overall quantities such as aerodynamic forces and wake size using the point measurement techniques such as laser doppler velocimetry (LDA) and pitot probes, Lienhart and Becker [2], Ahmed et al., [3]. Other works visualized the flow qualitatively using smoke, or oil to supplement their results (Azim and Gawad, [4]; Gilhome et al., [5]; Jenkins, [6]. Even though these techniques give sufficient information about the wake properties, the flow structures still remain far from being fully resolved.

Recently, a quantitative visualization using PIV (particle image velocimetry) has been tried to investigate the flow field pattern around a road vehicle. Cogotti and Gregorio, [7] studied the wake of therear-viewmirror and the flow field around the left side of a frontal wheel of the full-size vehicle. McCutcheon et al., [8] investigated the wake flow of the simplified car model according to the angle of the hatchback. Al-Garni and Bernal, [9] carried out PIV measurements to examine the characteristics of the turbulent flow around a lorry. However, these studies have not analyzed completely the whole wake including recirculating zone but done only a simple investigation on the flow features in the far wake. It is necessary to have a systematic approach Gushchin et al., [10] and Calluaud et al., [11] in the aspect of threedimensional vortex structures because the safety as well as the fuel consumption rate in driving a road vehicle is determined to a great extent by the highly three-dimensional flow characteristics around that.

In this study, PIV measurements are carried out for the analysis of two-dimensional vortices structures developed in the wake behind a passangers car, which generally adopts an air spoiler on the rear end to increase the driving stability in a high speed regiones. For the experiments, a vehicle model with/without rear air spoiler is set up in a closed wind tunnel facility and the velocity fields in the wake are measured in multiple sectional planes paralle to the streamwise direction.

\section{Experimental Setup:}

Fig.(1-a) indicates a layout of the PIV system and test section. The PIV system consists of the closed-loop wind tunnel, Nd-Yag laser, CCD camera, synchronizer and computer for the image pre-processing and post processing.

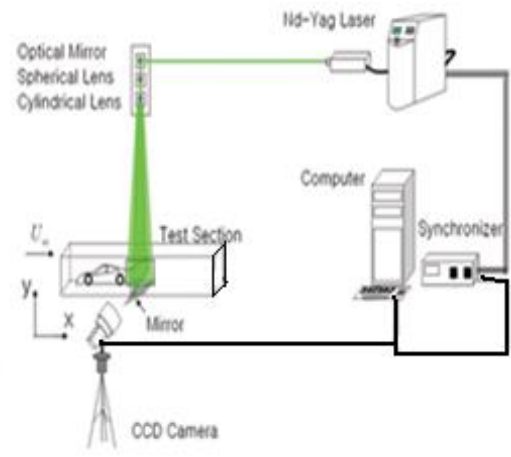

Fig.(1-a) Layout of test section and PIV system

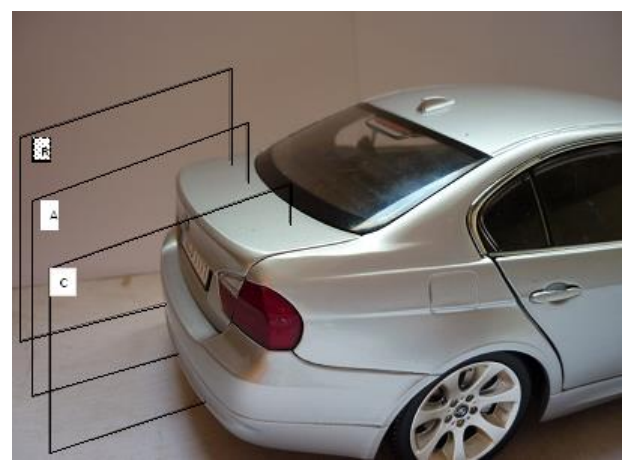

Fig. (1-b) Measurement planes behind a road vehicle 
The test section is made from a transparent acrylic are and with the dimensions, $52.4 \mathrm{mmX} 152.4 \mathrm{mmX} 450 \mathrm{~mm}$ respectively. The output energy of the Nd-Yag laser is $30 \mathrm{~mJ}$ with $532 \mathrm{~nm}$ wave length. The CCD camera has the resolution of $1280 \times 1024$ pixels. The thickness of light sheet is 1 $\mathrm{mm}$. the duration time between two laser pulses is calculating with aid of PIV calculator program, and taken between 17:190 $\mu$ s based on the rage of maximum to minimum used speed, which has the rang of $23: 2 \mathrm{~m} / \mathrm{s}$. The seeding particles are olive oil vapour. A model vehicle used is BMW series3, 320I with scale 1:18 from full size car. It was fixed at the center of the bottom surface of the test section.

Fig. (1-b), shows the cartesian coordinates system is referenced to determine the relative measuring positions of the vehicle from the symmetric plane. Each of the $\mathrm{x}, \mathrm{y}$ and $\mathrm{z}$ represents the axes for the direction of length $(\mathrm{L})$, height $(\mathrm{H})$ and width $(\mathrm{W})$ of the vehicle, respectively. During this plane measurement, a flat rectangular mirror is attached on the outside surface of the bottom of the transparent test section to illuminate the under part of an air spoiler and enough area behind the car rear end enable to investigate the flow pattern. The mirror which has a dimension of $20 \times 30 \mathrm{~mm} 2$ is attached at the bottom surface of the test section with the angle of $45^{\circ}$ to the horizontal plane at the downstream.

To observe the different wake flow features, two vehicle models were used; both have the same shape only except the existence of the air spoiler. At each sectional plane, 500 pairs of images were captured at a rate of $8 \mathrm{~Hz}$ by the CCD camera in order to investigate instantaneous flow structures and the velocity data were averaged over all the instantainous data. The focal length of the CCD camera lens is $600 \mathrm{~mm}$ and $\mathrm{x}-\mathrm{y}$ and $\mathrm{y}-\mathrm{z}$ plane measurement is 20.0 and $18.3 \mathrm{pixel} / \mathrm{mm}$, respectively. For the vector processing, a cross-correlation algorithm see Sung and Yoo, [12]. on FFT is applied using interrogation windows $32 \times 32$ pixels with $50 \%$ overlapping. To enhance the signal-to-noise ratio, a window shifting and cross-correlation technique is adopted.

In present study, a closed circuit design. Apart from superior flow quality control, the main factor in selecting a closed circuit arises from requirement specifications of the PIV system. Since this measurement technique requires the injection of seeding particles into the flow, it is desirable to contain these particles within an enclosed environment. It would be extremely difficult and wasteful to seed an open circuit tunnel as the seeding particles are continuously released to the atmosphere and therefore irrecoverable. Once the volume within a closed circuit tunnel is fully seeded, it will re-circulate without the need for continuous flow, so we will used the closed type wind tunnel as it specialist for PIV. The closed circuit type wind tunnel used for this study, utilizing air as its working fluid, and attaining a maximum test section speed of $23.7 \mathrm{~m} / \mathrm{s}$.

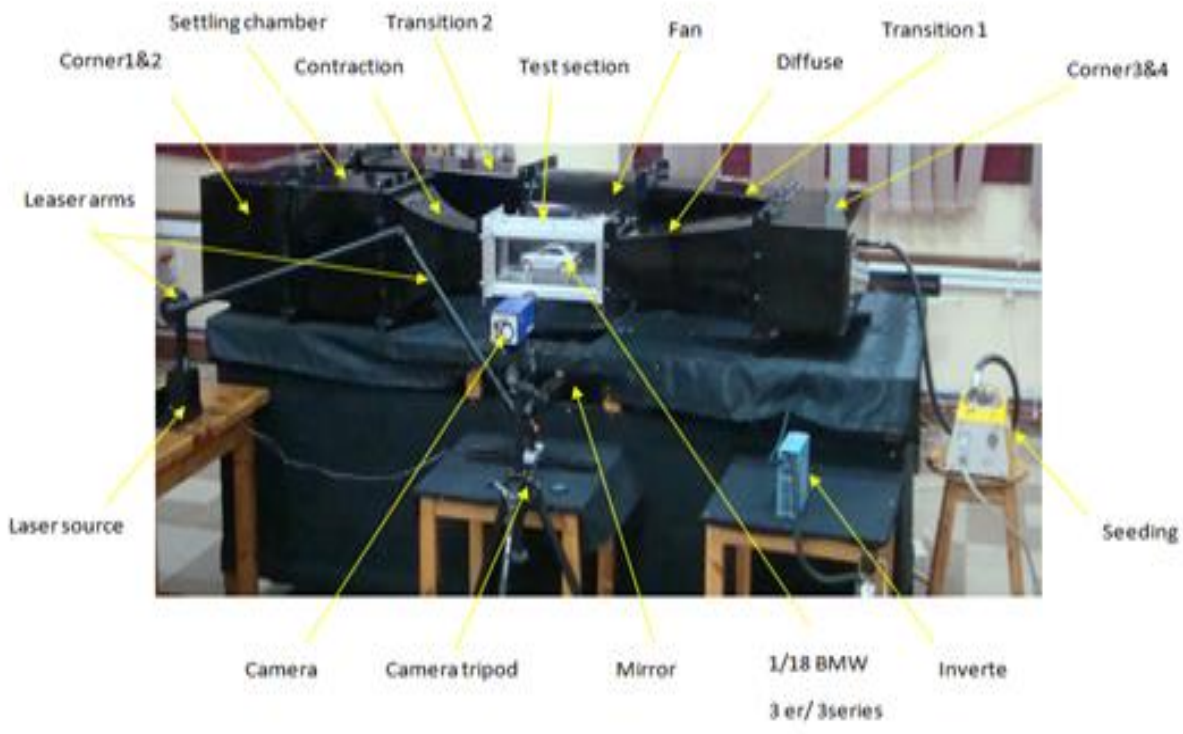

Figure (4) Photograph of the setup.

\section{Test Section}

The test section was where all aerodynamic measurements were to be performed since the tunnel was designed to be used with the optical measurement technique of PIV. It was logical to allow for the maximum optical access possible in the test section. For this reason, the test section was fabricated using transparent acrylic panels for four walls.

This would allow access for the laser and camera at any point around the test section, resulting in more convenient equipment setups. The four panels were fastened together using hex socket cap screws as opposed to adhesives in order to allow for access to the inside of the test section without moving any of the wind tunnel components.

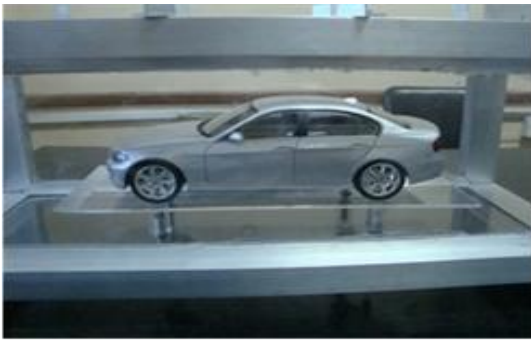

Figure (5-c) Car in test section

\section{Particle generation and supply:}

The use of natural seeding is sometimes acceptable, if enough visible particles are naturally present to act as markers for PIV. It is desirable to add tracers in order to achieve sufficient images contrast and control particle size. In gas flows, as the present work, the supply of tracer is very often more critical for the quality and feasibility of the PIV measurement and the health of the experimentalists if they have to breath seeded air. A seeding generator in Fig., (14), was used in the present work to generate the seeding particles by using a compressed air with 1.5 - 2.5 bar pressure difference with respect to the outlet pressure and creates air bubbles within the liquid oil.

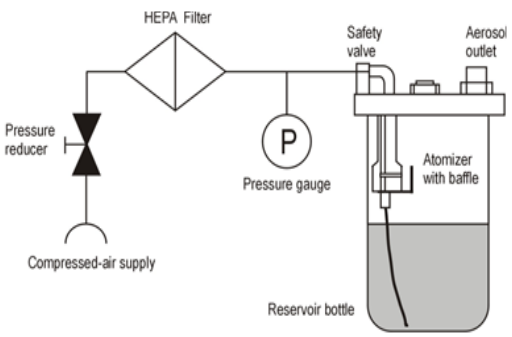

Schema of the ATM 230 
The particles can therefore not simply be supplied a long time before the measurements, but must be injected into the flow shortly before the gaseous medium enters the test section. The injection has to be done without significantly disturbing the flow, but in a way and at a location that ensures homogenous distribution of the tracer. So the tracer particles inter wind tunnel. Vegetable oil is the most commonly used liquid since oil droplets are believed to be less unhealthy than many other particles. However, any kind of seeding particles, which cannot be dissolved in water, should not be inhaled. Most vegetable oils (except cholesterol-free oils) lead to polydisperse distributions with mean diameters of approximately 1 $\mu \mathrm{m}$. These particles offer the advantage of not being toxic; they stay in air at rest for hours, and do not change in size significantly under various conditions. Hence, olive oil is chosen, during this work, because of its lubricating action.

\section{Results and Discussion}

To be sure from the result of PIV system, first we measuring the flow through the wind tunnel without car

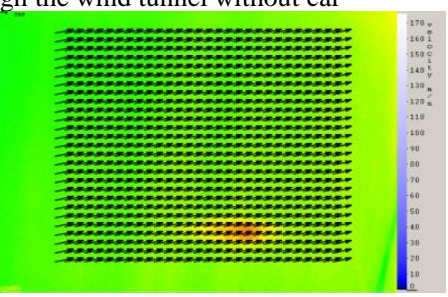

Fig, (15) flow through wind tunnel without car

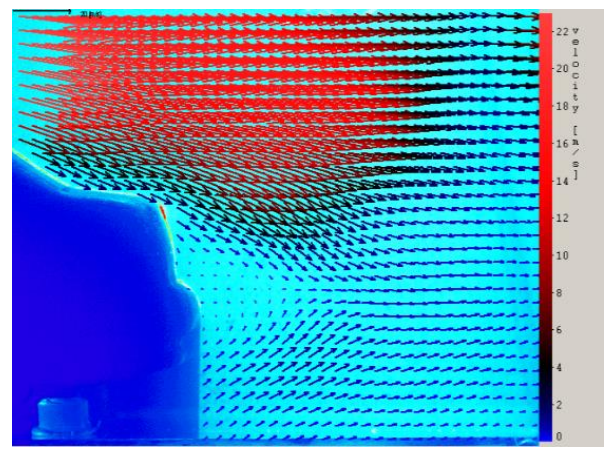

Velocity vector (wood car)

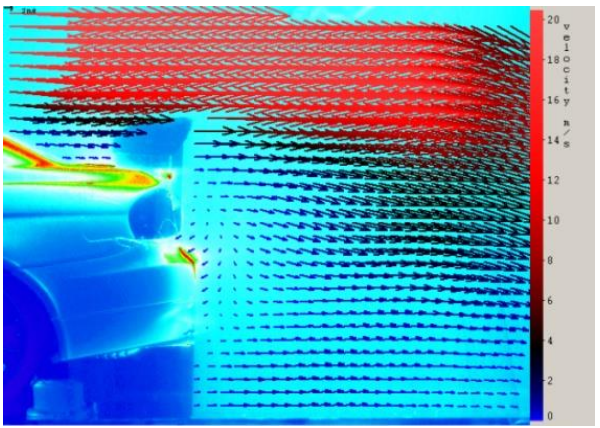

Velocity vector (BMW car with $6 \mathrm{~mm}$ spoiler)

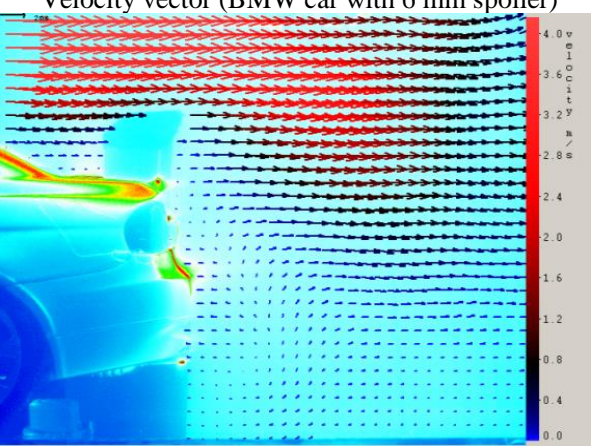

velocity vector (BMW car with $10 \mathrm{~mm}$ spoiler)
In this study, we measuring three model wood car (same dimensions of BMW car) BMW $3^{\text {rd }}$ series 320 scale 1:18 with/without spoiler. The measurements are taking in three planes $\mathrm{A}, \mathrm{B}$ and $\mathrm{C}$ as shown in Fig (1).

Prior to this study, the measurements in two different Reynolds numbers of $16.37 \times 10^{3}$ up to $3.77 \times 10^{5}$ based on the vehicle length were carried out and showed that there was little difference in view of flow structure between them. Accordingly, the result will be shown just for the case that the Reynolds number is $3.77 \times 10^{5}$. The aim of this research is confined to observe flow structures of the wake behind a road vehicle according to whether it has an air spoiler or not.

Comparing between the velocity vectors around the car in three cases and different height for the rear spoiler in plane A which is the symmetric plane of the car At $23.7 \mathrm{~m} / \mathrm{s}$ :

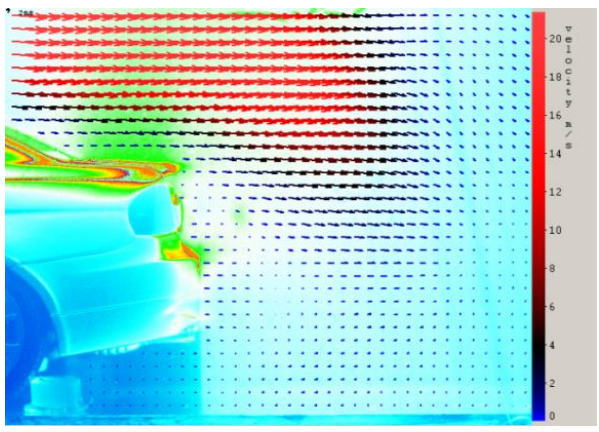

Velocity vector (BMW car without spoiler)

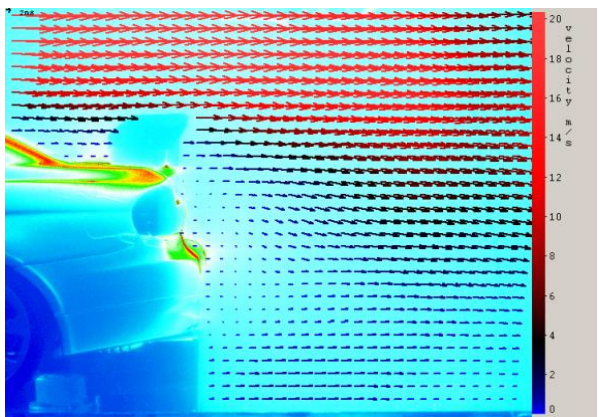

Velocity vector (BMW car with $8 \mathrm{~mm}$ spoiler)

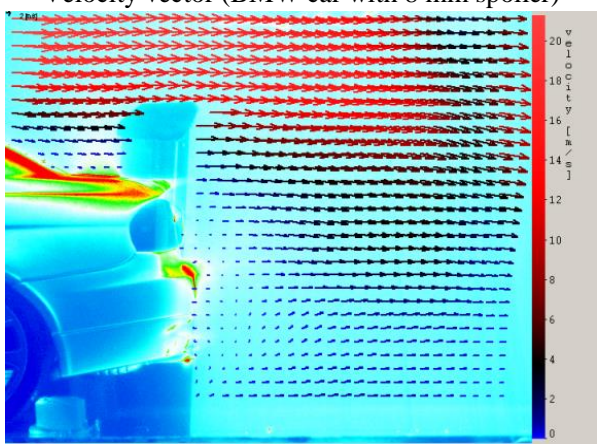

Velocity vector (BMW car with $12 \mathrm{~mm}$ spoiler)

Figure (16) Velocity vectors for different cases plane (A) 
Comparing between the streamlines around the car in three cases and different height for the rear spoiler in plane A which is the symmetric plane of the car At $23.7 \mathrm{~m} / \mathrm{s}$ :

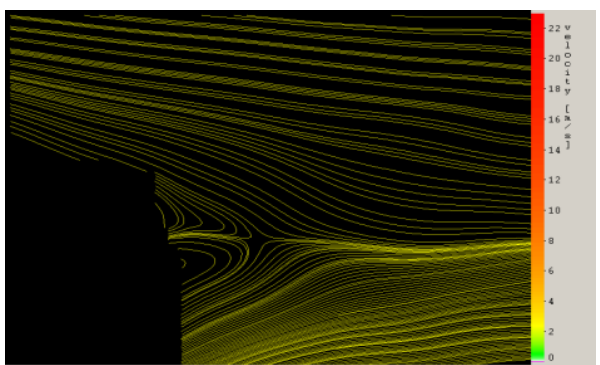

streamlines(wood car)

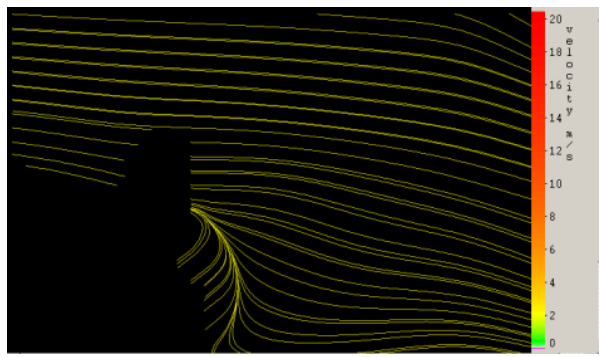

streamlines(BMW car with $6 \mathrm{~mm}$ spoiler)

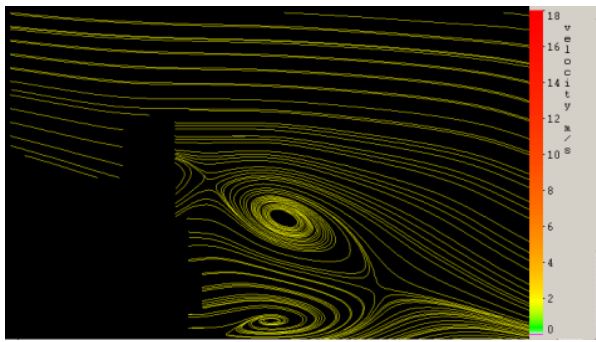

streamlines(BMW car with $10 \mathrm{~mm}$ spoiler)

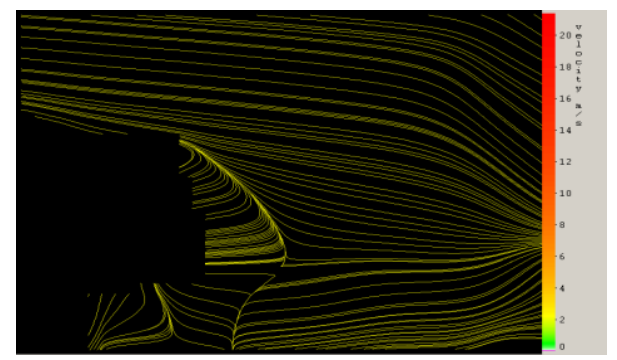

streamlines(BMW car without spoiler)

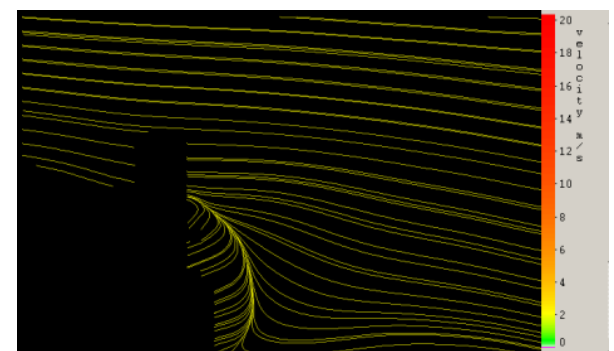

streamlines(BMW car with $8 \mathrm{~mm}$ spoiler)

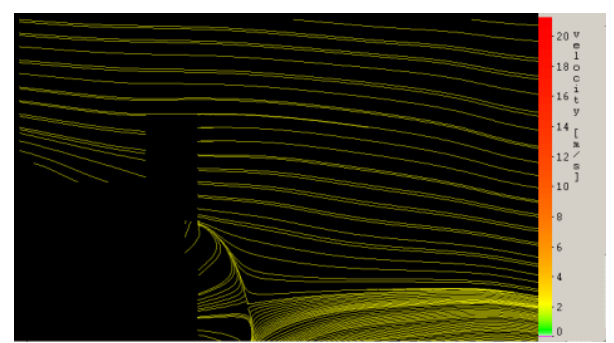

streamlines(BMW carwith 12mm spoiler)

Figure (17) streamlines for different cases plane (A)

Comparing between the velocity vectors around the car in three cases and different height for the rear spoiler in plane B which is the symmetric plane of the car At $23.7 \mathrm{~m} / \mathrm{s}$ :

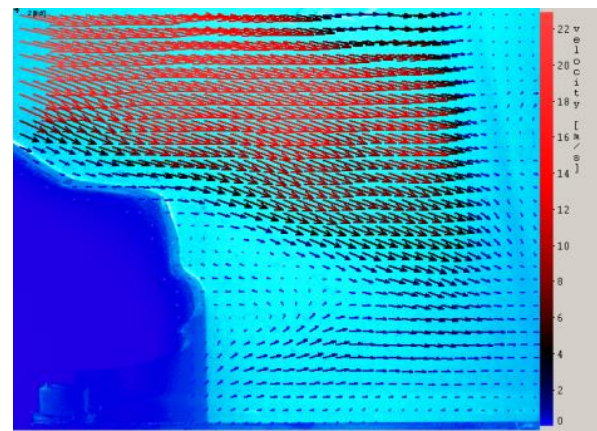

Velocity vector (wood car)

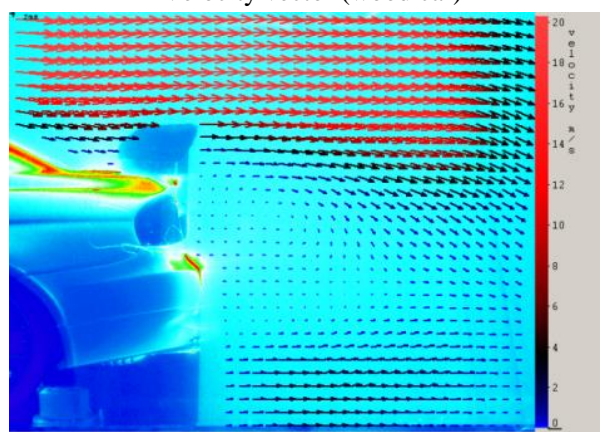

Velocity vector (BMW car with $6 \mathrm{~mm}$ spoiler)

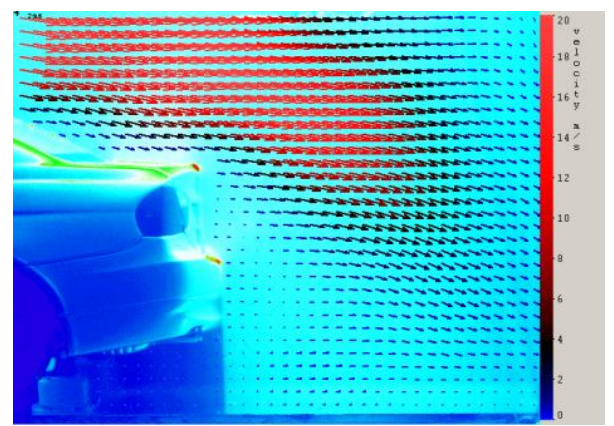

Velocity vector (BMW car without spoiler)

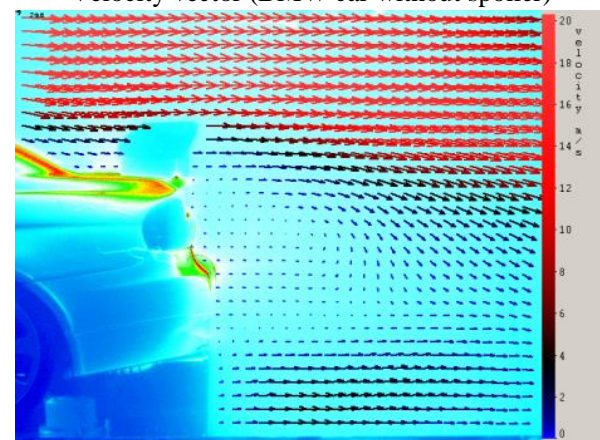

Velocity vector (BMW car with $8 \mathrm{~mm}$ spoiler) 


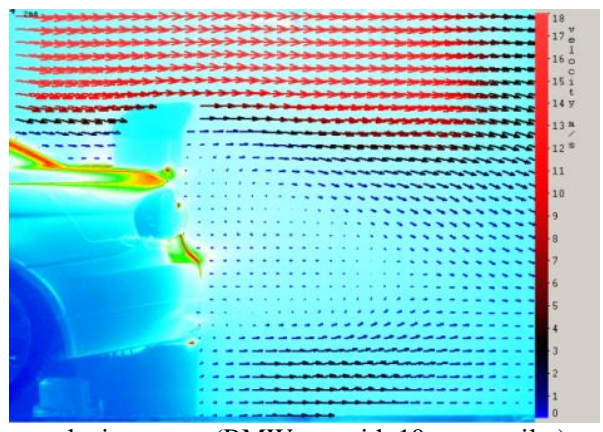

velocity vector (BMW car with $10 \mathrm{~mm}$ spoiler)

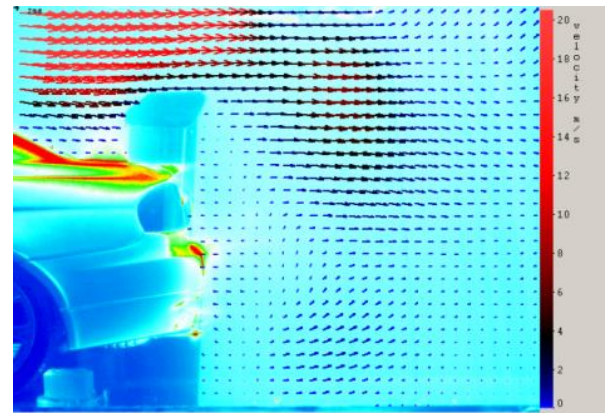

Velocity vector (BMW car with $12 \mathrm{~mm}$ spoiler)

Figure (18) Velocity vectors for different cases plane (B)

Comparing between the streamlines around the car in three cases and different height for the rear spoiler in plane B which is the symmetric plane of the car At $23.7 \mathrm{~m} / \mathrm{s}$ :

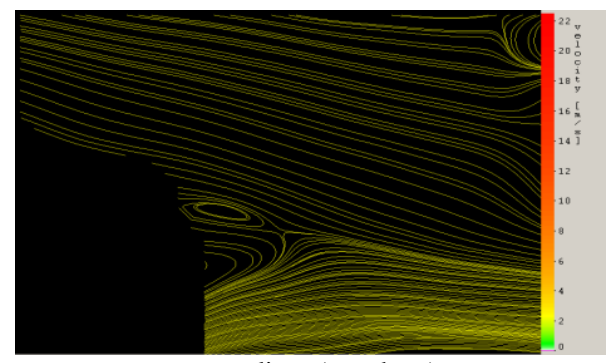

streamlines (wood car)

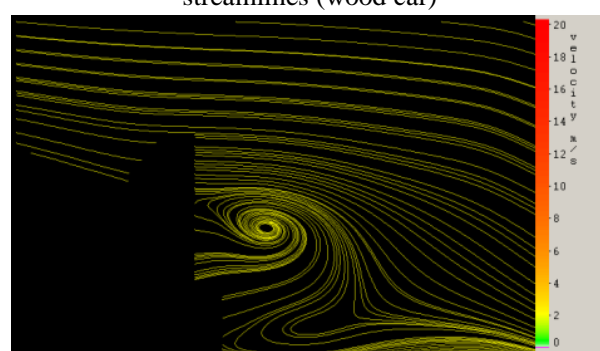

streamlines (BMW car with $6 \mathrm{~mm}$ spoiler)

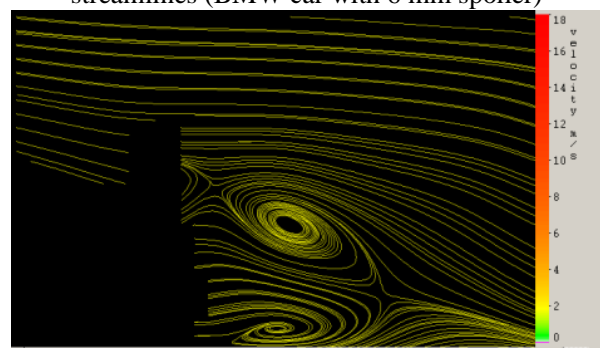

streamlines (BMW car with $10 \mathrm{~mm}$ spoiler)

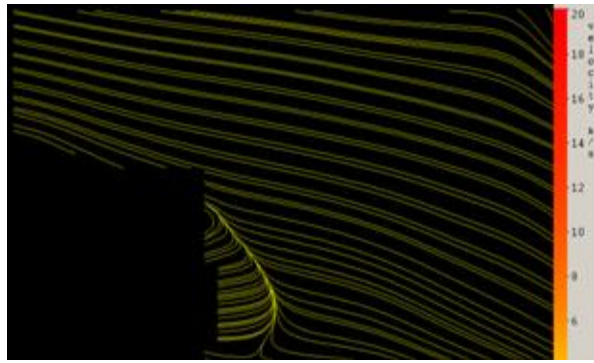

streamlines (BMW car without spoiler)

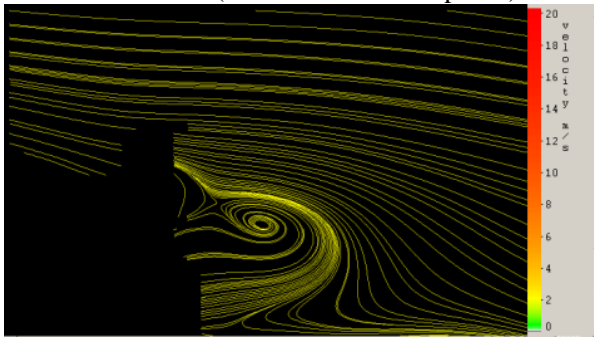

streamlines (BMW car with $8 \mathrm{~mm}$ spoiler)

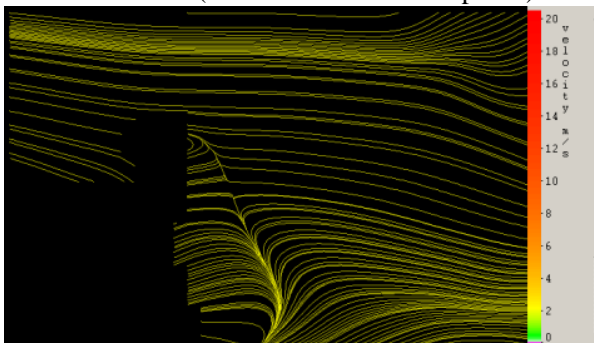

streamlines (BMW car with $12 \mathrm{~mm}$ spoiler) Figure (19) streamlines for different cases plane (B)

After measurement we found plane B same result of plane C that we have same vortex and streamlines patterns.

\section{Case study}

We consider the car with spoiler height $10 \mathrm{~mm}$ as the base case study to compare it with the other different cases.

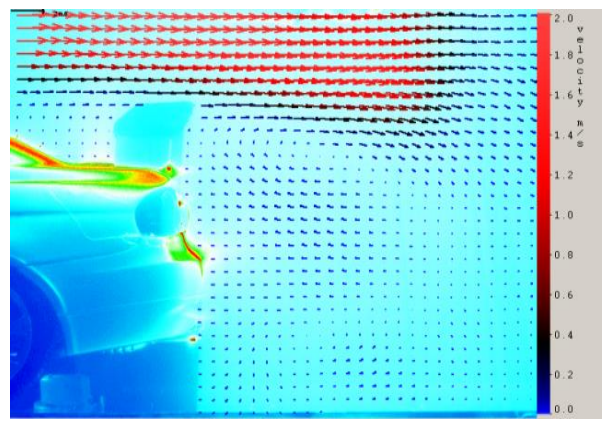

Velocity vector at speed $2.15 \mathrm{~m} / \mathrm{s}$
Velocity vectors for a car with spoiler height $10 \mathrm{~mm}$ at different velocities in plane (B):

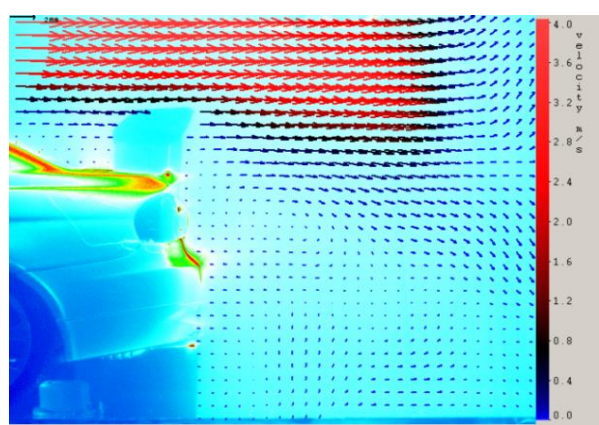

Velocity vector at speed $4.35 \mathrm{~m} / \mathrm{s}$ 


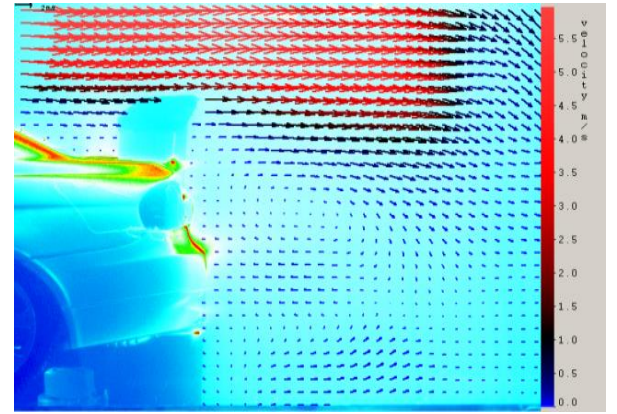

Velocity vector at speed $6.5 \mathrm{~m} / \mathrm{s}$

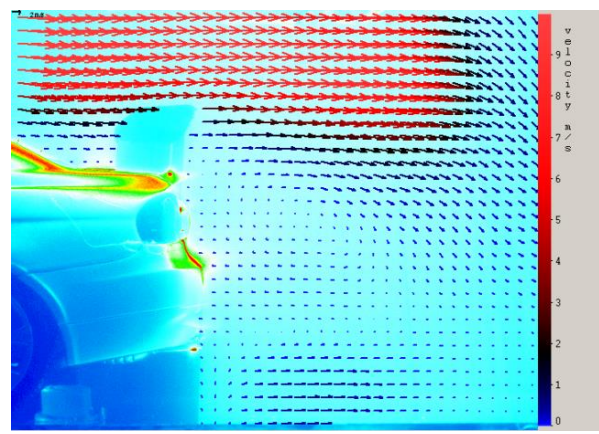

Velocity vector at speed $10.2 \mathrm{~m} / \mathrm{s}$

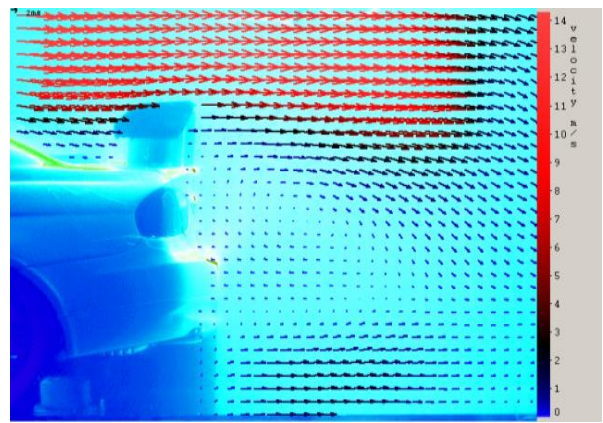

Velocity vector at speed $15.4 \mathrm{~m} / \mathrm{s}$ Figure (20) Velocity vector for car with spoiler $10 \mathrm{~mm}$ height plane (B), at different speeds

Streamlines for a car with spoiler height $10 \mathrm{~mm}$ at different velocities in plane (B):

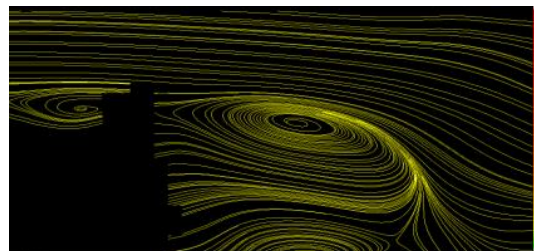

streamlines at speed $2.15 \mathrm{~m} / \mathrm{s}$

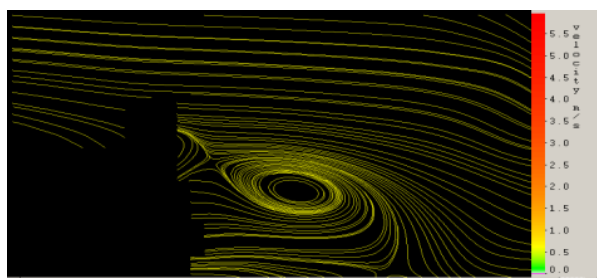

streamlines at speed $6.5 \mathrm{~m} / \mathrm{s}$

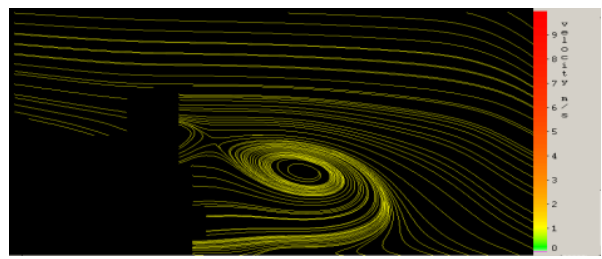

streamlines at speed $10.2 \mathrm{~m} / \mathrm{s}$

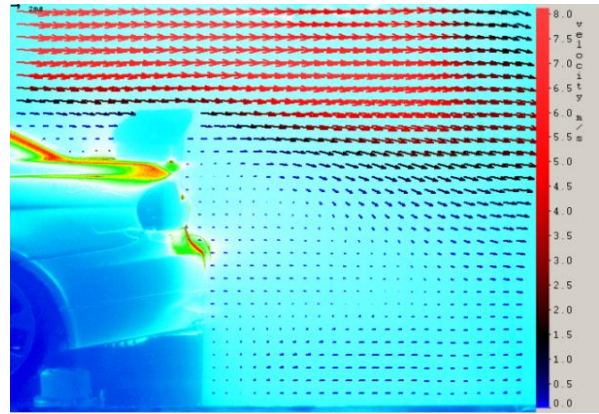

Velocity vector at speed $8.9 \mathrm{~m} / \mathrm{s}$

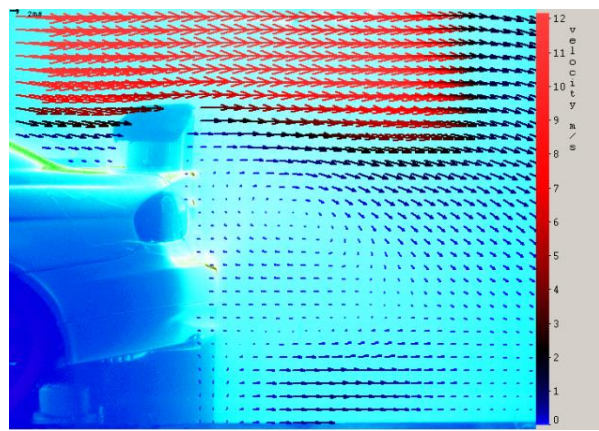

Velocity vector at speed $12.7 \mathrm{~m} / \mathrm{s}$

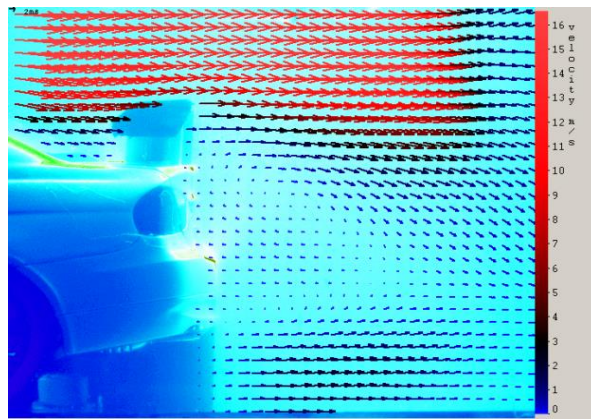

Velocity vector at speed $\quad 18.2 \mathrm{~m} / \mathrm{s}$

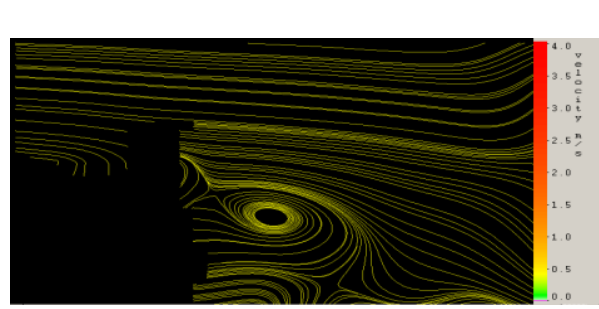

streamlines at speed $4.35 \mathrm{~m} / \mathrm{s}$

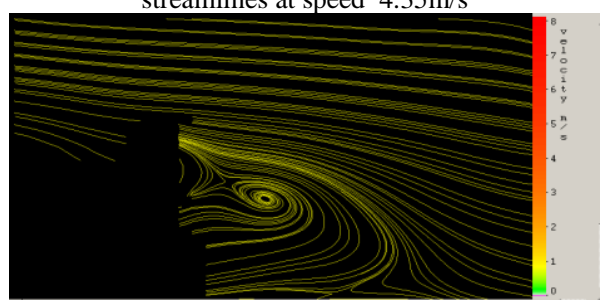

streamlines at speed $8.9 \mathrm{~m} / \mathrm{s}$

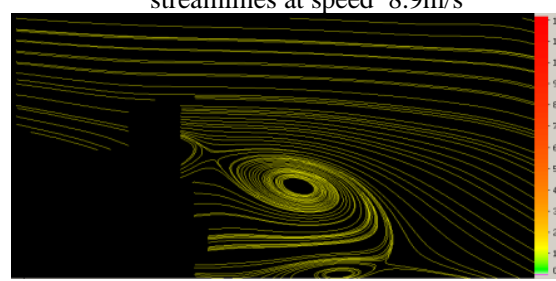

streamlines at speed $12.7 \mathrm{~m} / \mathrm{s}$ 


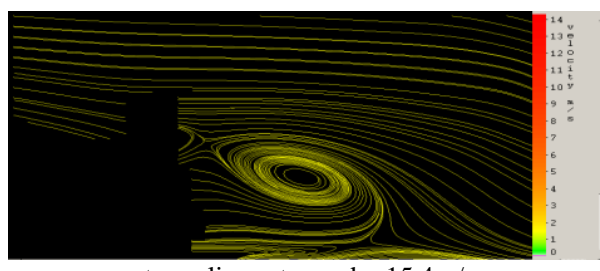

streamlines at speed $15.4 \mathrm{~m} / \mathrm{s}$

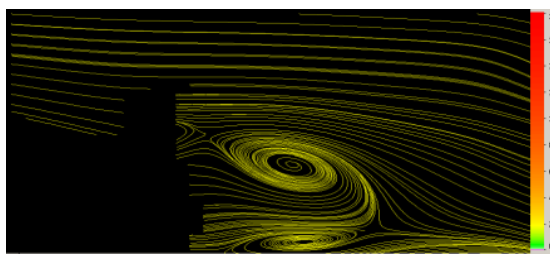

streamlines at speed $20.9 \mathrm{~m} / \mathrm{s}$

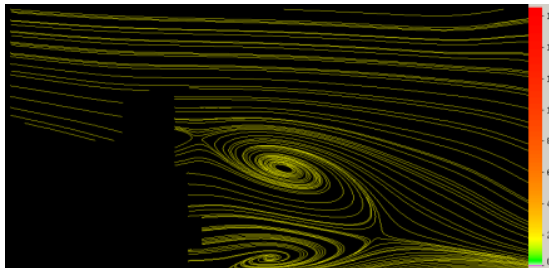

streamlines at speed $18.2 \mathrm{~m} / \mathrm{s}$

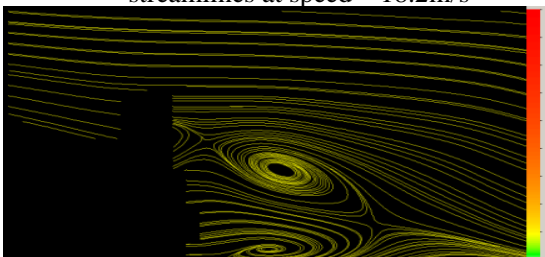

streamlines at speed $23.7 \mathrm{~m} / \mathrm{s}$

Figure (21) streamlines for car with spoiler $10 \mathrm{~mm}$ height plane (B), at different speeds

The turbulence intensity increase as the car speed increase as shown in figure 22 and figure 23

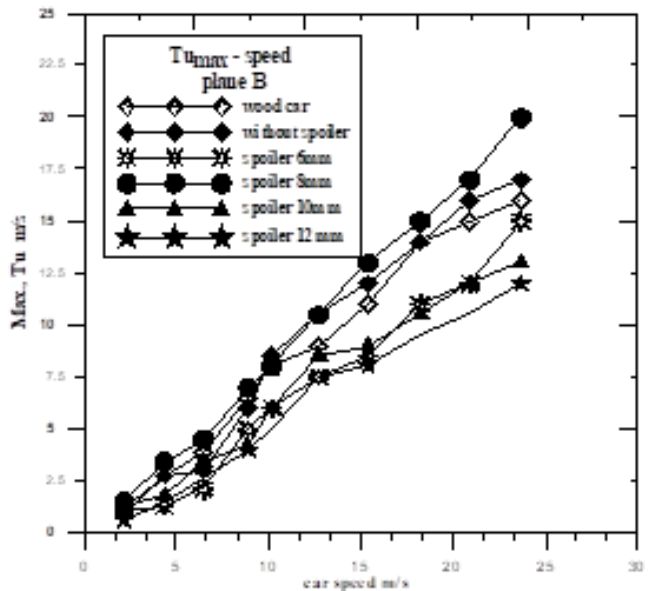

Figure (22) Relation between max., turbulence intensity and car speed plane A

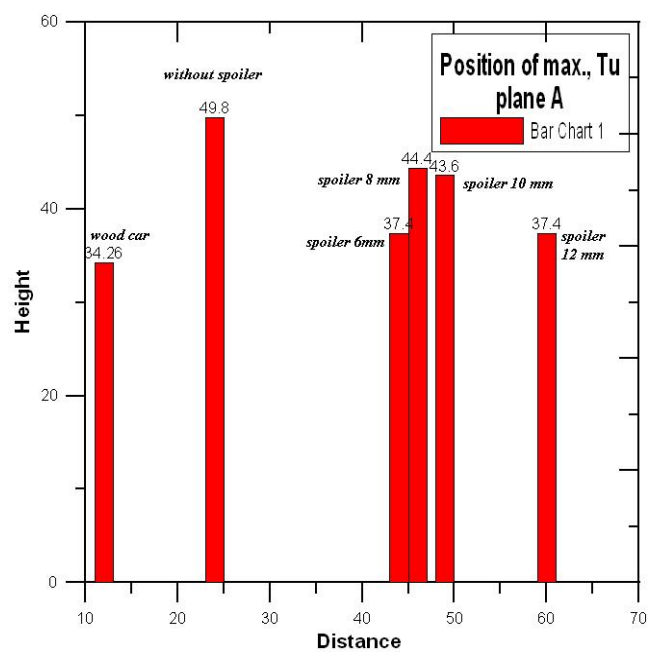

Figure (24) Position of max., Turbulence intensity in plane A
Position of maximum turbulence intensity shown in figure 24 and figure 25

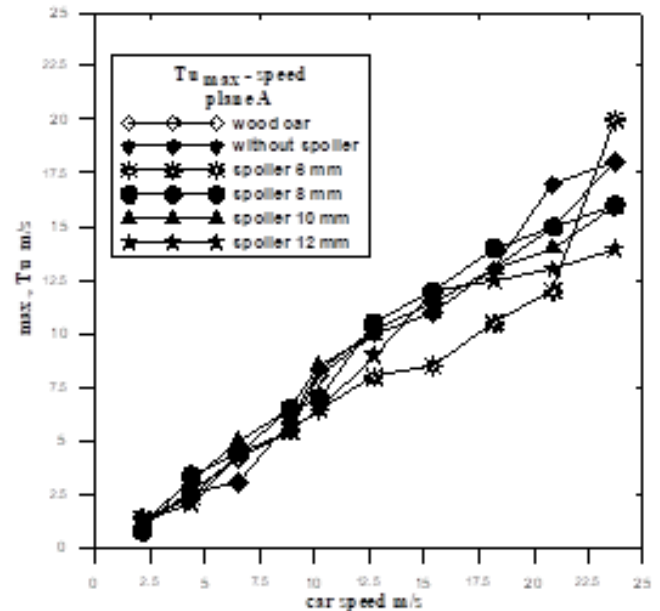

Figure (23) Relation between max., turbulence intensity and car speed plane B

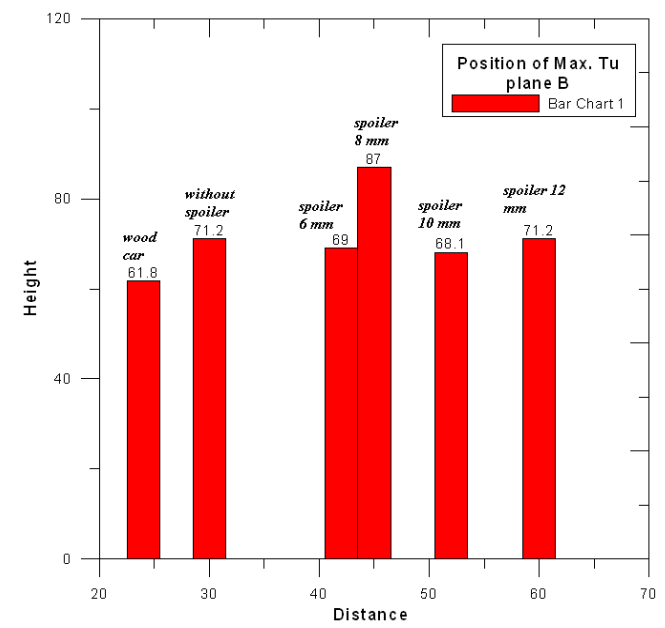

Figure (25) Position of max., Turbulence intensity in plane B 
The recirculation zone formed larger in the height direction near the symmetric plane and narrower to the width one. The movements of the focus showed different patterns from each other. The one goes downward and while the other moves upstream as the flow field is observed toward outside from the center plane of the vehicle. The stream wise wing tip vortices induced by an air spoiler have opposite rotational direction to the generated vortices. These wing tip vortices are an important factor to enhancement driving stability. Also, there found an interesting feature that node of the stream wise vortices prevents strong reverse flow of the span wise vortex from being formed in the lateral side because it stays near the location of the saddle point of the span wise vortex.

\section{Conclusions}

With the PIV measurement technique, experiments have been carried out to visualize complicated two-dimensional vortex motions and to examine the effect of an air spoiler on the wake of the road vehicle at the Reynolds number in the range of $16.77 \times 10^{3}$ up to3.77 $\times 10^{5}$.

\section{References}

[1]- Krajnovic, S. and Davidson, L., Large-Eddy Simulation of the Flow Around Simplified Car Model, SAE Paper, 2004-01-0227 (2004).

[2]- Ahmed, S. R., Ramm, G. and Faltin, G., Some Salient Features of the Time-Averaged Ground Vehicle Wake, SAE Paper, 840300 (1984).

[3]- Lienhart, H. and Becker, S., Flow and Turbulence structure in the Wake of a Simplified Car Model, SAE Paper, 2003-01-0656 (2003)
[4]- Azim, A. F. A. and Gawad, A. F. A., A Flow Visualization Study of the Aerodynamic Interference Between Passenger Cars, SAE Paper, 2000-01-0355 (2000)

[5]- Gilhome, B. R., Saunders, J. W. and Sheridan, J., Time Averaged and Unsteady Near-Wake Analysis of Cars, SAE Paper, 2001-01-1040 (2001)

[6]- Jenkins, L. N., An Experimental Investigation of the Flow Over the Rear End of a Notchback Automobile Configuration, SAE Paper, 2000-01-0489 (2000)

[7]- Cogotti, A. and De Gregorio, F., Presentation of Flow Field Investigation by PIV on a Full-Scale Car in the Pininfarina Wind Tunnel, SAE Paper, 2000-01-0870 (2000).

[8]- McCutcheon, G., McColgan, A. H., Grant, I. and Hurst, D., Wake Studies of a Model Passenger Car Using PIV, SAE Paper,2002-01-3335 (2002).

[9]- Al-Garni, A. M. and Bernal, L. P., Experimental Investigation of the Near Wake of a Pick-up Truck, SAE Paper, 2003-01-0651 (2003).

[10]- Gushchin, V. A., Kostomarov, A. V. and Matyushin, P. V., 3D Visualization of the Separated Fluid Flows, Journal of Visualization, 7-2 (2004), 143-150

[11]- Calluaud, D., David, L. and Texier, A., Vortex Shedding Process Investigation Downstream a Surface-Mounted Block, Journal of Visualization, 8-2 (2005), 99-108.

[12]- Sung, J. and Yoo, J. Y., Three-Dimensional Phase Averaging of Time-Resolved PIV Measurement Data, Meas. Sci. Technol., 12 (2001), 655-662. 